\title{
Dynamic Channel Allocation Techniques Using Adaptive Modulation and Adaptive Antennas
}

\author{
Jonathan S. Blogh, Peter J. Cherriman, and Lajos Hanzo, Senior Member, IEEE
}

\begin{abstract}
This contribution studies the impact of adaptive quadrature amplitude modulation (AQAM) on network performance when applied to a cellular network, using adaptive antennas in conjunction with both fixed channel allocation (FCA) and locally distributed dynamic channel allocation (DCA) schemes. The performance advantages of using adaptive modulation are investigated in terms of the overall network performance, mean transmitted power, and the average network throughput. Adaptive modulation allowed an extra $51 \%$ of users to be supported by an FCA 4-QAM network, while in conjunction with DCA, an additional $54 \%$ user capacity was attained.
\end{abstract}

Index Terms-Adaptive antennas, adaptive modulation, adaptive arrays, beam-steering, DCA, dynamic channel allocation, .

\section{INTRODUCTION}

D YNAMIC channel allocation (DCA) schemes, in general, offer substantially improved new call blocking, call dropping, and grade-of-service (GOS) performance, when compared to fixed channel allocation (FCA) algorithms. In previous work, it was shown [1] that the locally optimized least interference algorithm (LOLIA), based upon the locally optimized DCA algorithms proposed by Priscoli et al. ([2] and [3]) provided the best overall compromise in terms of network performance.

The LOLIA always assigns the least interfered channel, which is not used within the nearest $n$ cells by another user, i.e., the LOLIA will select the channel with the lowest level of interference, which is not used by the nearest $n$ cells. Therefore, the higher the value of $n$, the greater the minimum reuse distance and hence more calls are blocked since there are less available channels, which are not used by the nearest $n$ base stations. Fig. 1 shows the LOLIA's frequency reuse "exclusion zone" set to seven base stations, i.e., $n=7$, and illustrates how the six neighboring cells and the user's current cell form the "exclusion zone" of the channel used in the center cell. Thus, unlike for the FCA algorithm, where the channel allocations, and hence the reuse distances, are fixed, the reuse constraint of the LOLIA is based around a user's current base station, and therefore "travels" with the user from base station to base station, limiting the number of channels available for allocation at the currently used base station. This dynamically located reuse constraint is removed, when the call is ended, unlike in FCA where the fixed frequency reuse constraints are permanent.

Manuscript received March 29, 1999; revised September 12, 2000.

The authors are with the Department of Electronics and Computer Science, University of Southampton, Southampton, U.K. (e-mail: jsb97@ecs.soton.ac.uk; 1h@ecs.soton.ac.uk).

Publisher Item Identifier S 0733-8716(01)00116-0.

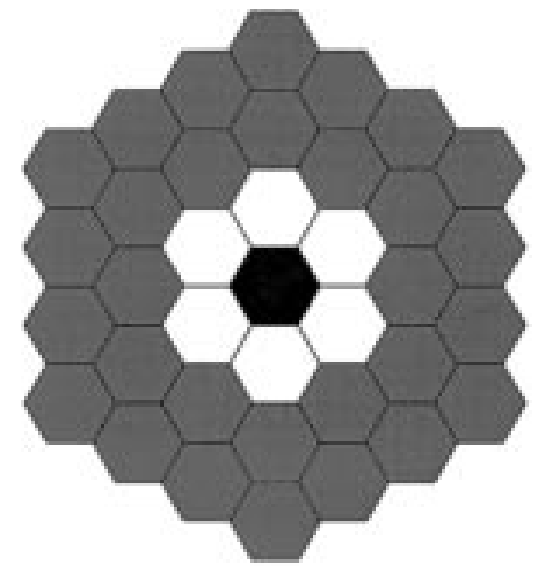

Fig. 1. Nearest-neighbor constraint for $n=7$ for the LOLIA showing the central cell in which the user is located (black) and the neighboring cells (white), in which the given channel may not be reused. However, the cells outside this "exclusion zone" (grey) may use this given channel.

Previous work [4], [5] focused on improving the network performance by using adaptive antennas at the base stations, and power control in both the up- and the down-links. This contribution builds upon this earlier work by the addition of adaptive quadrature amplitude modulation (AQAM) [6]-[8], as will be discussed in Section III. The paper is structured as follows. The next two sections describe the principles behind adaptive antennas, AQAM, and power control. Section IV introduces our simulation conditions, performance metrics, and the network parameters. Section V briefly characterizes the performance of a 4-QAM-based benchmark network, before engaging in a more detailed study of the results obtained using AQAM. Our conclusions are summarized in Section VI.

\section{ADAPTIVE ANTENNAS}

An adaptive antenna is constituted by an array of antenna elements, whose relative phases and amplitudes can be controlled, in order to allow strong reception of signals from a given direction, while attenuating interfering signals from other directions [9]. Since a cellular network is typically interference limited, adaptive antennas, sited at cellular base stations, are ideally suited for application in this field [9]-[12].

Given that each mobile in the network, or within a certain radius of the base station, is uniquely identifiable, the so-called sample matrix inversion (SMI) algorithm ([11], [13], and [14]) may be invoked to obtain the antenna array receiver weights, optimized with respect to the received signal-to-interference plus noise ratio (SINR) [14]. In order to calculate the receiver antenna array weights using the SMI algorithm, an eight-symbol 
long BPSK reference signal was assigned to the desired mobile, irrespective of the AQAM mode used for the data transmission. The remaining seven orthogonal eight-symbol BPSK reference signals were then assigned to the interfering mobiles, with these codes allocated to more than one mobile, if the number of interferers was higher than seven. Thus, with the desired mobile being uniquely identifiable, the receiver antenna weights optimized for obtaining the maximum received SINR, may be calculated.

The receiver antenna array weights calculated for use on the up-link may not be suitable for the down-link due to the generally uncorrelated up- and down-link channels of frequency division duplexing (FDD). However, using a feedback loop from the mobile to the base stations would allow the base station to use the down-link weights maximizing the SINR as proposed in [15] and [16]. This solution is also applicable to time division duplexing (TDD). Furthermore, the required antenna weights can be signaled jointly with the required AQAM modes.

\section{AdAPtiVE Modulation AND POWER CONTROL}

The idea behind AQAM is to select a modulation mode according to the instantaneous radio channel quality [17]. Since most practical and performance aspects of AQAM were detailed in [17]-[20], due to lack of space here only a brief overview is provided. Specifically, if the channel exhibits a high instantaneous SINR, then a high order modulation mode may be employed by the adaptive modem, enabling the exploitation of the temporarily high channel capacity. Likewise, if the channel has a low SINR, using a high order modulation mode would result in an unacceptable frame error rate (FER), and hence a more robust but lower throughput modulation mode would be invoked. Hence, AQAM not only combats the effects of a poor quality channel, but also attempts to maximize the throughput, while maintaining a given target FER. Thus, there is trade-off between the mean FER and the data throughput, which is governed by the modem mode switching thresholds. These switching thresholds define the SINRs, at which the channel is considered unsuitable for a given modulation mode, where an alternative mode must be invoked.

To elaborate a little further on the practical aspects of burst-by-burst AQAM modems, the transceiver's implementation has to satisfy the tighter specifications of the most demanding AQAM mode, say 16 QAM, in terms of clockand carrier-recovery as well as channel equalization [17]. This is the price paid for its robust, yet high-throughput performance. In simple terms, the modem can be viewed as an intelligent scheme, which momentarily drops the number of bits per AQAM symbol transmitted in anticipation of a burst of transmission errors in the high-throughput mode. Hence, its throughput is almost as high, as that of say the 16-QAM mode, while maintaining almost as high a robustness, as BPSK. The effects of co-channel interference on AQAM modems were detailed in [18], while the practical engineering aspects its latency- and buffering requirements, as well as the impact of various Doppler frequencies were studied in [19]. As a means of mitigating the effects of latency, frequency hopping was shown to be a powerful countermeasure.

In this context, there are two dominant factors influencing the AQAM system's performance [19]. As expected, the channel quality estimates delivered by the receiver to the remote transmitter, in order to instruct it as to the receiver's perception of the required AQAM mode for its next transmission burst are more up-to-date, when the Doppler frequency is low. This is a simple consequence of the slowly varying fading envelope. However, ironically, the buffering requirements are aggravated by low Doppler frequencies, since it takes a long time for the mobile to emerge from a deep fade, before it can deplete its buffer by invoking for example the high-rate 16-QAM mode. In [20], the mean squared error (MSE) at the output of the AQAM channel equalizer was found to be a reliable channel quality measure, although the bit error rate (BER) estimate of the channel decoder can also be successfully employed in this context. The signaling of the AQAM modes requires only two bits per transmitted frame, but these bits have to be strongly protected against corruption. We found that the best channel codes for such short messages were constituted by simple repetition codes decoded with the aid of majority logic decisions (MLD), which did not significantly impinge on the throughput of the AQAM modem. These codes were designed to ensure that the AQAM mode signalling would reliably operate under channel conditions, where the AQAM modem's target integrity could be maintained. Again, readers interested in further details on AQAM are referred to [17]-[20].

The power control algorithm implemented attempted to independently adjust the mobile and base station transmit powers, such that the up- and down-link SINRs were within a given target SINR window. The employment of a target window avoided constantly increasing and decreasing the transmission powers, which could lead to potential power control instabilities within the network. Furthermore, the range of possible transmission powers is analogous to an inherent power control error plus the slow fading envelope.

The combination of power control with AQAM leads to several performance tradeoffs, which must be considered when designing the power control and modulation-mode switching algorithm. For example, the transmitted power could be minimized, which could result in either a high BER and a high throughput, or a low FER and a low throughput. Alternatively, the FER could be lowered, even at a high throughput, when tolerating high transmission powers.

The power control and modulation mode switching algorithm invoked in our simulations attempted to minimize the transmitted power, while maintaining a high throughput with a less than $5 \%$ target FER.

Fig. 2 shows the AQAM and power control decision tree when in the 4-QAM mode. The first step in the process is to determine the lower of the up- and the down-link SINRs. The next step is to determine, whether the BPSK modulation mode should be selected. The conditions for this to occur are that, either the lower SINR is below the 4-QAM call-dropping threshold or that it is below the 4-QAM call-reallocation threshold and the maximum possible transmission power is currently used. If the lower SINR is below the 4-QAM call-reallocation threshold, or the SINR 


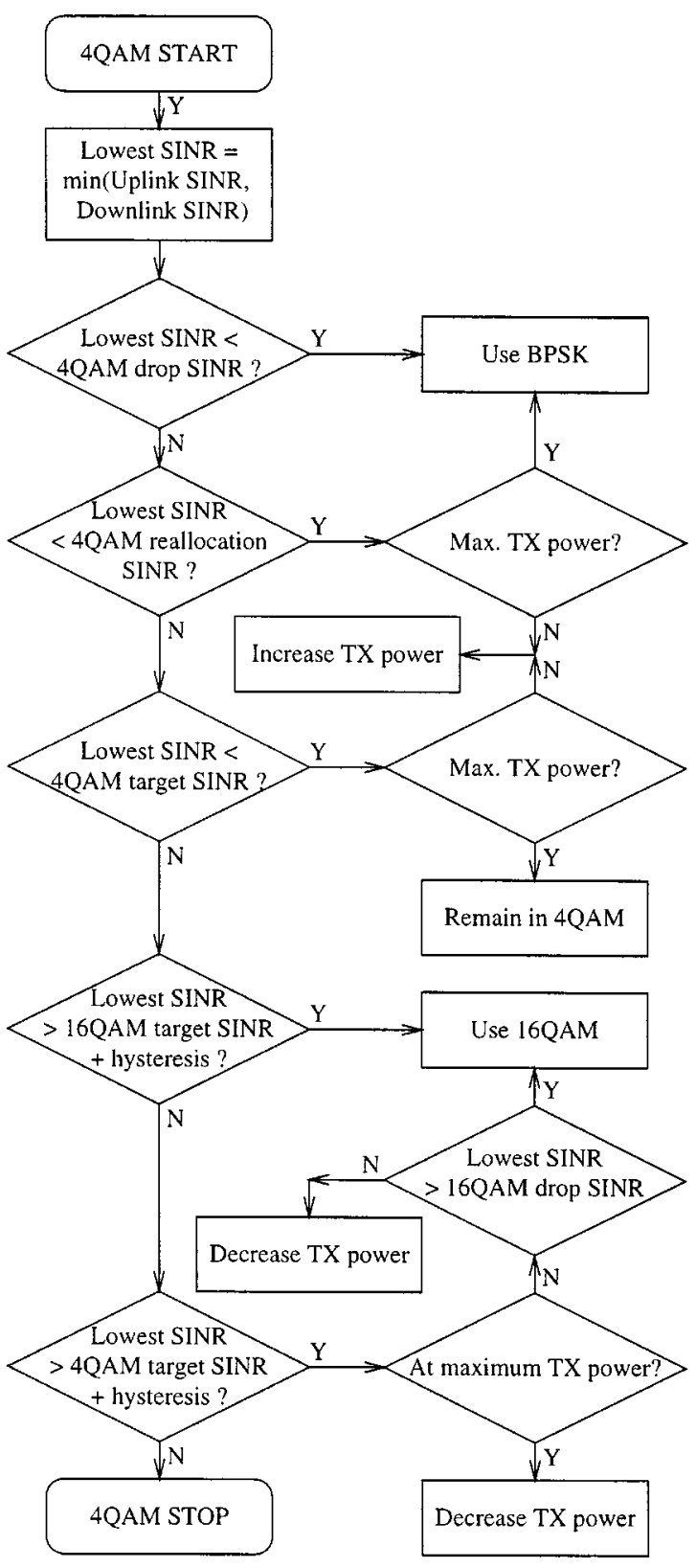

Fig. 2. AQAM mode and power control decision tree when in the 4-QAM mode.

is below the 4-QAM target SINR, and the maximum transmission power has not been reached, then the transmit power is increased. However, if the SINR is below the 4-QAM target SINR and the maximum possible transmit power is currently used, then the modem remains in the 4-QAM mode. The 16-QAM mode is chosen, if the SINR is higher than the 16-QAM target SINR, plus the associated hysteresis. Alternatively, the 16-QAM mode is invoked, if the SINR is higher than the 4-QAM target SINR plus the hysteresis, the transmission power required to obtain this SINR is not the maximum transmit power, and the SINR is higher than the 16-QAM call-dropping SINR. However, if the SINR is below the 16-QAM call-dropping SINR or the maximum transmission power is in use, then the transmit power is reduced to keep the SINR in the 4-QAM mode target SINR window. The improved SINR achieved through the use of adaptive antenna arrays at the base station permits a higher mean network data throughput.

However, if no beamforming is used, the multiple propagation paths would inflict a higher grade of intersymbol interference (ISI), which would increase the mean square error (MSE) at the output of the channel equalizer constituting the switching criterion of the AQAM modem. Hence, without beamforming, typically lower order modulation modes would be used due to the increased amount of ISI. Under the assumption of low propagation excess delay, predominantly narrowband Rayleigh fading conditions would prevail, although the similar-delay multipath components would exhibit different angle-of-arrival statistics, and therefore the effects of beamforming are still beneficial in terms of mitigating the multipath interference and hence enhancing the system's performance. The above two phenomena ultimately contribute toward the system's bits per symbol (BPS) throughput and enhance the received signal quality.

Let us now consider our simulation conditions.

\section{Simulation CONDITIONS}

\section{A. Performance Metrics}

There are several metrics that can be used for quantifying the performance or quality of service provided by a particular channel allocation algorithm. The following performance metrics have been widely used in the literature and were also advocated by [21].

- New call blocking probability $P_{B}$ is defined as the probability that a new call is denied access to the network. This may occur because there are no available channels at the desired basestation, or the available channels are subject to excessive interference.

- Call dropping or forced termination probability $P_{D}$ or $P_{F T}$ is the probability that a call is forced to terminate prematurely. This may be due to insufficient SINR, or there are no available channels of suitable quality to handover to on entering a new cell.

- Probability of low quality connection $P_{\text {low }}$ quantifying the chances that either the uplink or downlink signal quality is below the level ("reallocation SINR") required by the specific transceiver for maintaining a given target performance.

- Probability of outage $P_{\text {out }}$ is defined as the probability that the SINR value is below the threshold ("outage SINR"), where the call is deemed to be in outage.

- GOS was defined by [21] as

$$
\begin{aligned}
\mathrm{GOS}= & P\{\text { unsuccessful or low-quality call accesses }\} \\
= & P\{\text { call is blocked }\}+P\{\text { call is admitted }\} \\
& \times P\{\text { low signal quality and call is admitted }\} \\
= & P_{B}+\left(1-P_{B}\right) P_{\text {low }} .
\end{aligned}
$$

A handover or handoff event occurs, when the quality of the communications channel used degrades, and hence the call is switched to a newly allocated channel. If the new channel belongs to the same base station, this is referred to as an intracell handover. Generally an intracell handover is performed, when 
TABLE I

SIMULATION PARAMETERS

\begin{tabular}{l|r||l|r}
\hline Parameter & Value & Parameter & Value \\
\hline \hline Noisefloor & $-104 \mathrm{dBm}$ & Multiple Access & FDMA/TDMA \\
Frame duration & $0.4615 \mathrm{~ms}$ & Cell radius & $218 \mathrm{~m}$ \\
BS transmit power & $10 \mathrm{dBm}$ & MS transmit power & $10 \mathrm{dBm}$ \\
BS power control & $\mathrm{No}$ & MS power control & No \\
Number of basestations & 49 & Handover hysteresis & $2 \mathrm{~dB}$ \\
Outage SINR threshold & $17 \mathrm{~dB}$ & Re-allocation SINR threshold & $21 \mathrm{~dB}$ \\
Modulation scheme & $4 \mathrm{QAM}$ & Pathloss exponent & -3.5 \\
Number of timeslots & 8 & Number of carriers & 7 \\
Average inter-call-time & $300 \mathrm{~s}$ & Max new-call queue-time & $5 \mathrm{~s}$ \\
Average call duration & $60 \mathrm{~s}$ & Reference signal modulation & BPSK \\
Beamforning algorithm & $\mathrm{SMI}$ & Reference signal length & $8 \mathrm{bits}$ \\
MS speed & $30 \mathrm{mph}$ & Number of antenna elements & 2,4 \& 8 \\
Pathloss it. 1m reference point & $0 \mathrm{~dB}$ & Shadow fading & No \\
\hline
\end{tabular}

the channel quality degrades due to co-channel interference or in order to increase the system's performance and capacity. Intercell handovers occur typically, when the mobile approaches the fringes of the cell area, and hence the signal strength degrades, requiring a handover to a nearer basestation.

For a network employing power control and adaptive modulation, it is also beneficial to monitor the transmission powers used, and the proportion of time that each of the modulation schemes is activated, thus allowing the average modem throughput to be determined.

\section{B. System Parameters}

A microcellular system, having the parameters defined in Table I, was simulated in order to investigate the performance of adaptive antenna arrays in a system employing 4-QAM with and without power control, and AQAM using power control, for both FCA and LOLIA DCA schemes [2], [3]. The maximum capacity of the system was limited through the use of eight timeslots and just seven carrier frequencies, in order to maintain an acceptable computational load. If a channel allocation request for a new call could not be satisfied immediately, it was queued for up to $5 \mathrm{~s}$. If not serviced during this time period, it was classed as blocked. The base stations comprising the network were assumed to be synchronized, hence preserving perfect orthogonality between different timeslots. The call duration and intercall periods were Poisson-distributed [22], [23] with the mean values shown in Table I. The user activity rate was set fairly high, in order to expedite our simulations. The mobiles were free to move in fixed random directions, at a speed of $30 \mathrm{mi} / \mathrm{h}$ within the simulation area which consisted of 49 cells, each with a radius of $218 \mathrm{~m}$. Simulating a "desert island"-type simulation area can result in the central cells experiencing harsher co-channel interference levels than those at the edges of the simulation area, leading to overly optimistic network performance results. Thus, a tessellating rhombic simulation area of $7 \times 7$ cells was used, in order to allow the simulation area to be replicated around itself. In other words, the mobile stations and their signals and interferences are "wrapped around" from one side of the network to the other. For example, a mobile station in call which crosses the edge of the network, re-enters the network on the diametric edge, while inflicting CCI to all users which may be located at either edge of the network.

Three parameters were used to model the physical layer, the "outage SINR," the "reallocation SINR" and the "target SINR." When the up- or down-link SINR for a given mobile drops below the "reallocation SINR," then the mobile requests a new physical channel to handover to, initiating an intra- or intercell handover. If the signal quality drops below the "outage SINR," then an outage occurs. A prolonged outage leads to the call being dropped. The "target SINR" is the SINR to be maintained by the power control algorithm.

The "reallocation SINR" and the "outage SINR" are defined as the average SINR's necessary for a 5\% and $10 \%$ transmission frame error rate (FER), respectively, using a given modulation mode (BPSK, 4-QAM and 16-QAM). The SINR levels necessary to obtain these FERs were evaluated via simulation [24] for each of the possible modulation modes over a narrowband Rayleigh fading channel, for approximately half-rate Bose-Chaudhuri-Hocquenghem (BCH) codes, which employed interleaving over the different number of bits conveyed by the different modem modes. The "target SINR" was chosen to maximize the network capacity and represents an FER of approximately $2 \%$.

Since a dropped call is less desirable from the user's point of view than a blocked call, a handover queueing system (HQS) was employed. A HQS forms a queue of handover requests, which have precedence over new calls for network resources, hence reducing the number of dropped calls at the expense of the new call blocking probability. An additional benefit of HQS is that a user may be held in the handover queue, for a limited time, until network resources are freed, thus increasing its chances of a successful handover. 
The calculation of the receive antenna array weights was performed on a frame-by-frame basis, leading to updated up- and down-link SINRs every frame. These SINR values were then used to select the modulation mode and transmission power to be employed, and to determine whether any channel re-allocation was necessary. Hence, frame-by-frame adaptive modulation, power control, and dynamic channel allocation was performed.

The multipath propagation environment considered consisted of a line-of-sight (LOS) ray and two additional rays, each having a third of the power of the LOS ray, with angles of arrival at the basestation determined using the so-called geometrically based single-bounce elliptical model (GBSBEM) of [25] and [26] with parameters chosen such that the multipath rays had one third of the received power of the direct ray. It was assumed that all of these multipath rays arrived with zero time delay relative to the LOS path, or that a space-time equalizer [27], [28] was employed, thus making full use of the additional received signal energy. However, the many extra desired and interfering signals incident upon the antenna array rapidly consume the limited degrees of freedom of the antenna array, limiting its ability to fully cancel each source of interference.

A further assumption was that the up- and down-link channels were identical, thus allowing the same antenna pattern to be used in both the up- and the down-links, as in a TDD system. This assumption of channel reciprocity should therefore give an upper limit to the performance gains that may be achieved using an adaptive array.

\section{Performance Study}

\section{A. Performance of a 4-QAM-Based Network Using Power Control}

Our investigations commenced with the comparison of the FCA and LOLIA DCA schemes in networks with and without power control. The fixed channel allocation scheme employed a seven-cell reuse cluster, corresponding to one carrier frequency per base station. The LOLIA was used in conjunction with a frequency reuse exclusion zone of seven nearest base stations, i.e., $n=7$. The immediate effect of power control can be seen in Fig. 3. This figure shows that the power control attempts to maintain a constant SINR, which is sufficiently high for reliable communications across the network, rather than providing unnecessarily high SINRs near the base station and insufficient levels of SINR far from the base stations. This is evident for a network without power control in Fig. 3. The high SINR values of the power-controlled scenario near the base station are due to the transmitter's inability to reduce its transmitted power below its minimum threshold.

The effect of power control on the mean number of handovers performed per call becomes clear from Fig. 4. From this figure, it can be seen that with respect to the number of handovers per call for the FCA algorithm, the performance of the network with power control significantly exceeded that of the network without power control using an adaptive antenna array of twice the number of antenna elements. The use of power control in conjunction with the FCA algorithm led to a mean reduction in the number of handovers by a factor of 3.7. The inherently good

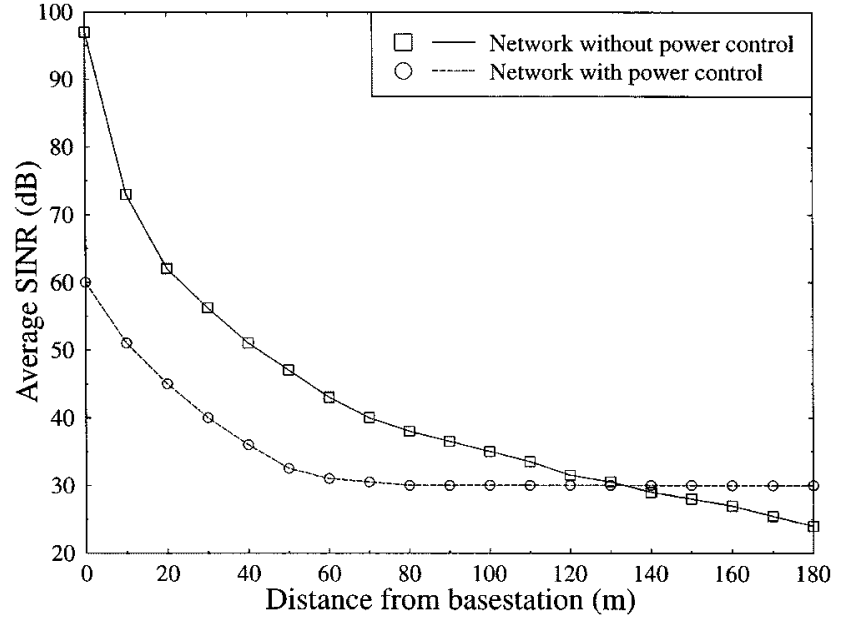

Fig. 3. Average SINR against distance from the base station for networks with and without power control using the parameters of Table I.

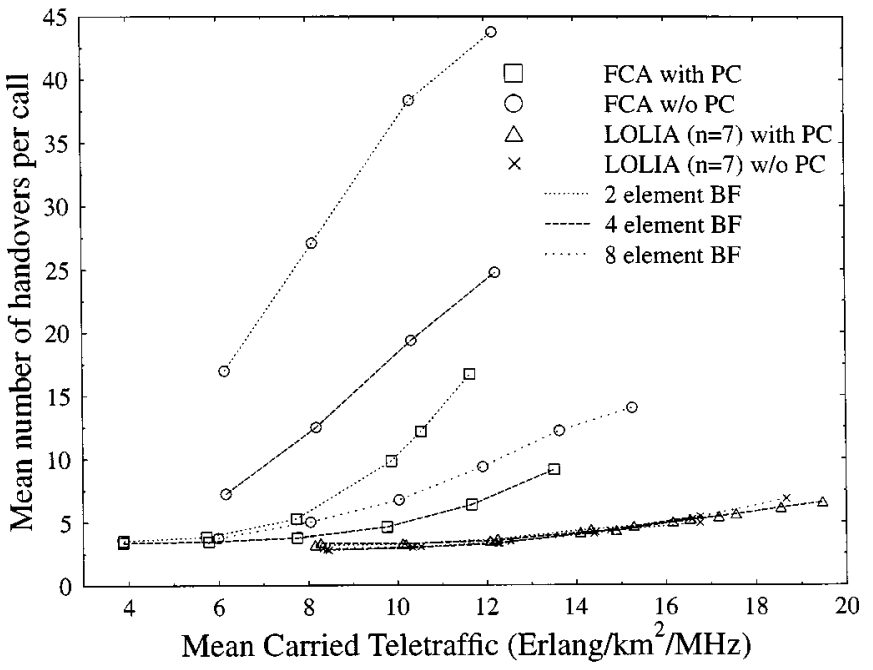

Fig. 4. Mean number of handovers per call versus mean carried traffic of the LOLIA, with seven "local" base stations, and of FCA employing a seven-cell reuse cluster, for two- and four-element antenna arrays, with and without power control using the parameters of Table I.

performance of the LOLIA was also slightly improved on average.

Although not shown explicitly, the new call-blocking probability for the FCA algorithm deteriorated slightly through the use of power control as a direct consequence of the improved call-dropping probability shown in Fig. 5. The new call-blocking performance of the LOLIA was superior to that of the FCA algorithm both with and without power control, as a result of the dynamic nature of the LOLIA. This enables the LOLIA to allocate any of the available channels not used within the seven-cell exclusion zone (maximum of 56 in this scenario) to a new call request. However, the FCA algorithm only has one carrier frequency per base station, and therefore is less likely to be able to satisfy a new call request. The addition of power control to the LOLIA with $n=7$ led to a reduced new call-blocking probability. Specifically, the new call-blocking probability with power control is reduced to that achieved using twice the number of antenna elements without power control. The higher new call-blocking probability for the 


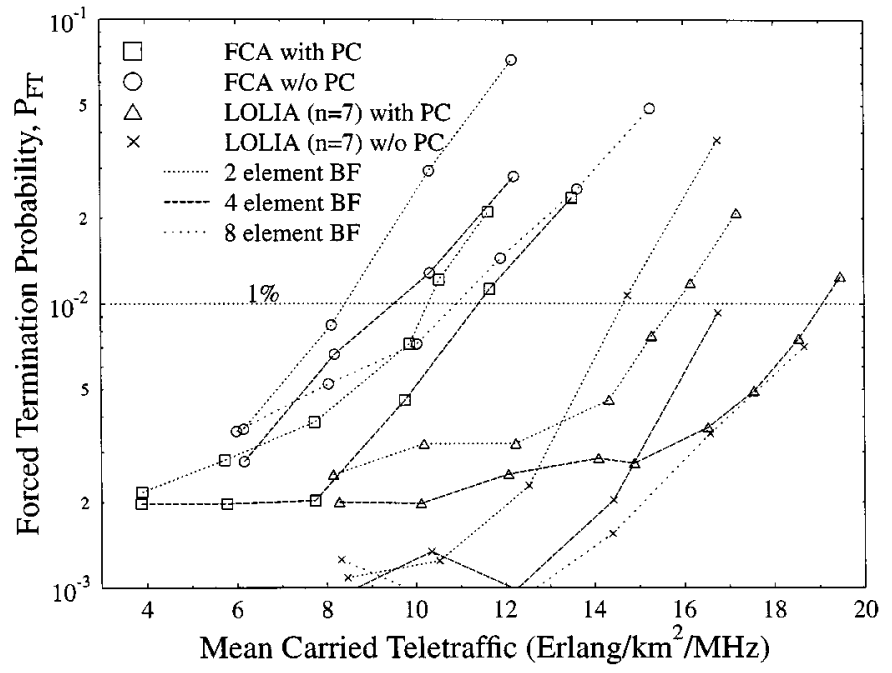

Fig. 5. Call-dropping probability versus mean carried traffic of the LOLIA, with seven "local" base stations, and of FCA employing a seven-cell reuse cluster, for two- and four-element antenna arrays, with and without power control using the parameters of Table I.

network without power control can be attributed to the lower SINR values, which prevent new call initiation, whereas the higher average SINR levels of the network with power control enables additional calls to commence.

Fig. 5 shows that the call-dropping probability is significantly reduced for both the FCA algorithm and the LOLIA with $n=$ 7. The FCA with power control offers a lower call-dropping probability, than a similar network without power control using twice the number of adaptive antenna elements. However, at traffic loads of below approximately seven Erlangs $/ \mathrm{km}^{2} / \mathrm{MHz}$, the call-dropping probability begins to level off for the FCA algorithm. This phenomenon is particularly noticeable in conjunction with the LOLIA and results from the power control algorithm limiting the maximum SINR, leading to a flatter call-dropping profile than that of the network without power control. Thus, at lower traffic loads, the network without power control has a higher average SINR, leading to less dropped calls. However, at higher levels of teletraffic, the power control algorithm offers a lower call-dropping rate, as a consequence of the lower levels of interference present using the power control scheme.

A further advantage of using power control in a cellular mobile network is portrayed in Fig. 6, which shows that the mean transmit power is significantly reduced from the fixed transmit power of $10 \mathrm{dBm}$ due to power control. The mean transmit powers for both the FCA algorithm and the LOLIA are significantly reduced, with the four-element adaptive antenna arrays offering a further $0.5-1.5 \mathrm{~dB}$ reduction in the transmit power over that of the two-element arrays.

Having characterized the performance of our 4-QAM benchmark scheme, let us now consider our proposed AQAM system.

\section{B. Performance of an AQAM-Based Network Using Power Control}

This section presents the simulation results obtained for a network using burst-by-burst adaptive modulation [17]-[20] invoked in order to improve the network performance. Simulations were conducted for both a standard seven-cell FCA

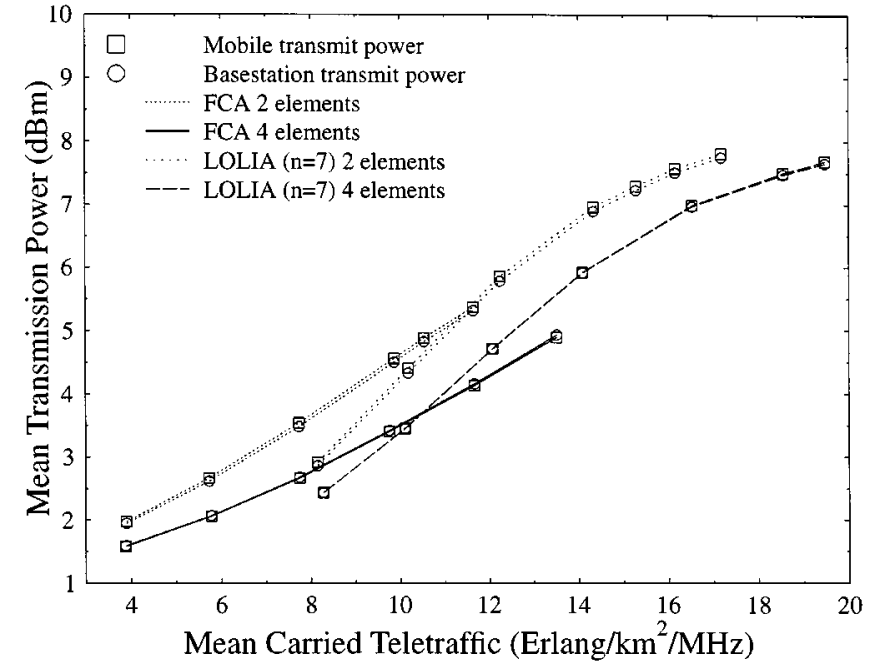

Fig. 6. Mean transmit power versus mean carried traffic of the LOLIA, with seven "local" base stations, and of FCA employing a seven-cell reuse cluster, for two- and four-element antenna arrays, with and without power control using the parameters of Table I.

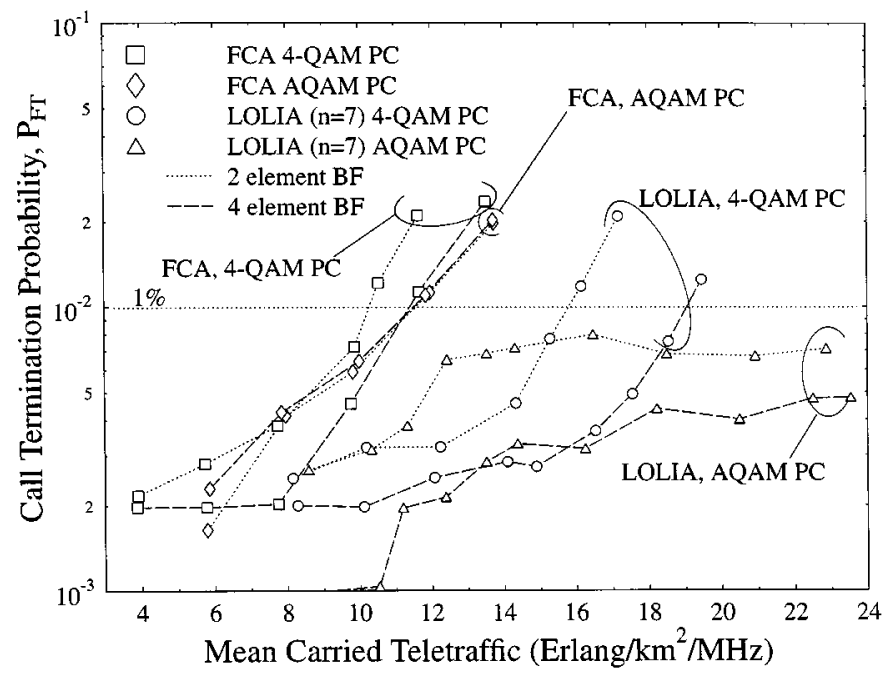

Fig. 7. Call-dropping, or forced-termination, performance versus mean carried traffic of the LOLIA, with seven "local" base stations, and of FCA employing a seven-cell reuse cluster, for two- and four-element antenna arrays, with and without AQAM.

scheme and a seven-cell LOLIA system. The results obtained for a four-QAM-based network using power control were included for comparison purposes.

The new call-blocking probability (not shown) was only marginally changed for the FCA algorithm using power control with four-QAM or AQAM, suggesting that the new call-blocking performance of the FCA algorithm was limited by the lack of available frequency-timeslot combinations, rather than by inadequate signal quality. This analysis was confirmed by the improvement in the new call blocking performance of the LOLIA resulting from the improved signal quality of AQAM.

The call-dropping probability is depicted in Fig. 7. The AQAM LOLIA using $n=7$ had, in general, a reduced call-dropping probability compared to that of four-QAM with power control due to the resilience of AQAM in poor-quality signal conditions. The dropped calls were caused exclusively by 


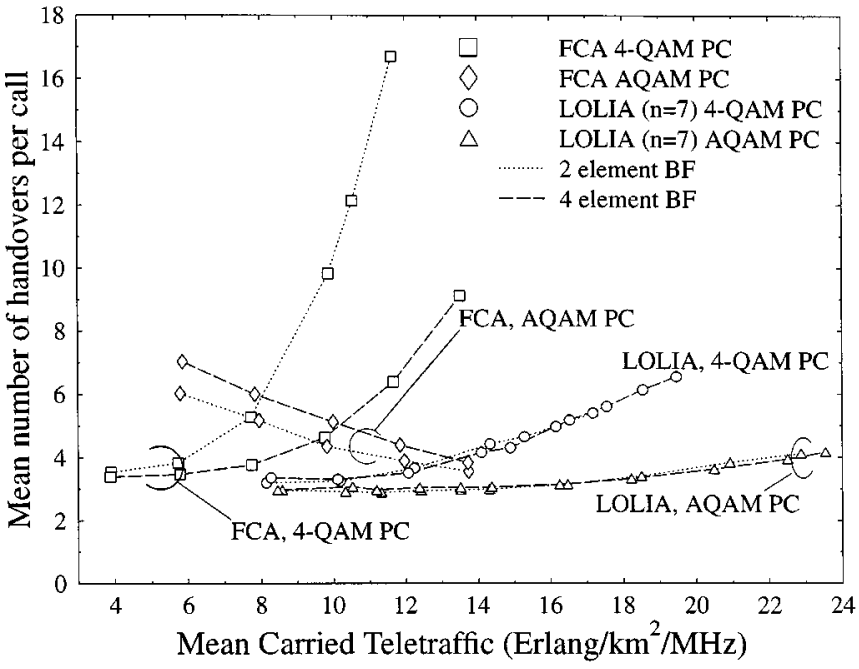

Fig. 8. Mean number of handovers per call versus mean carried traffic of the LOLIA, with seven "local" base stations, and of FCA employing a seven-cell reuse cluster, for two- and four-element antenna arrays, with and without $A Q A M$.

insufficient signal quality during the intracell handover process, thus increasing the number of adaptive antenna elements from two to four improved the call-dropping performance. The higher dropping probability observed for traffic loads between 10.5 and 15 Erlangs $/ \mathrm{km}^{2} / \mathrm{MHz}$ when using the two-element adaptive antenna array was due to the power control and AQAM attempting to trade off modem throughput and transmit power, while attempting to minimize the number of dropped calls. The extra interference suppression capabilities of the four-element adaptive antenna array led to a reduced call-dropping probability. Hence, altering the AQAM mode selection algorithm (in Fig. 2), may improve its performance at these traffic loads when used with a two-element antenna array.

At low levels of traffic, the FCA dropped the majority of its calls during intracell handovers. However, as the network loading increased, the proportion of calls dropped in the intercell handover process increased significantly due to the lack of available slots. The four-element adaptive antenna array reduced the number of dropped calls due to the intracell handover procedure, but the corresponding rise in the number of calls dropped during intercell handover resulted in an overall degradation in the call-dropping performance. Therefore, the call-dropping performance using the FCA algorithm is limited by the availability of frequency-timeslot combinations during handovers, and the results using AQAM are bounded by those of four-QAM in conjunction with power control.

Fig. 8 shows the mean number of handovers per call for four-QAM and AQAM, both using power control. The LOLIA with $n=7$ performed a lower total number of handovers per call when using AQAM due its inherent resilience to poor signal quality conditions.

The breakdown of the handovers into intercell and intracell handovers is given in Fig. 9 and shows that the extra interference rejection capabilities, and the associated superior SINR, of the four-element array results in a lower number of intracell handovers for the LOLIA. Since the intracell handover process is the primary cause of dropped calls, and less intracell handovers are performed when using a four-element antenna, more inter-

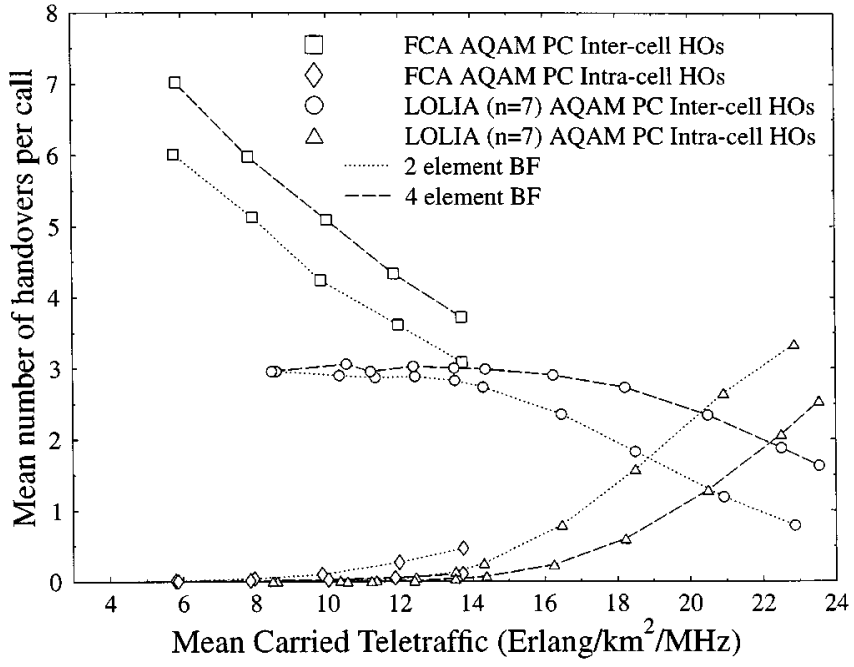

Fig. 9. Mean number of intercell and intracell handovers per call versus mean carried traffic of the LOLIA, with seven "local" base stations, and of FCA employing a seven-cell reuse cluster, for two- and four-element antenna arrays, with AQAM.

cell handovers are necessitated in the network using four-element adaptive antenna arrays, as the users roam from cell to cell.

In contrast, the number of intracell handovers performed in conjunction with the FCA algorithm decreases, as the teletraffic rises, and the number of antenna elements is increased from two to four. This is a consequence of the particular implementation of the modulation mode selection-power control algorithm and its interaction with the FCA handover process. The AQAM algorithm attempts to remain in the current modulation mode as long as possible, and hence as the SINR degrades will opt for performing an intracell handover in an attempt to maintain the SINR, rather than drop to a lower order modulation mode suitable for the reduced SINR level. Thus, with a four-element adaptive antenna array, the average (and instantaneous) SINR is higher than that of a two-element array, leading to a more frequent usage of the less resilient higher order modulation modes, which requires additional intracell handovers. However, as the mean teletraffic increases, so does the level of interference in the network and a greater proportion of transmission time is spent in the lower order modulation modes, thus requiring less intracell handovers, as illustrated in Fig. 9.

The probability of a low quality (LQ) access is depicted in Fig. 10, showing an interesting interaction between the FCA algorithm and the AQAM implementation. The probability of a LQ access occurring is reduced, as the traffic level increases and the number of antenna elements is decreased. This can be attributed to the less frequent usage of the higher order modulation modes at the higher traffic loads, hence the lower order modulation modes are used more frequently and thus the chance of a LQ access taking place is reduced. The four-element adaptive antenna array leads to a higher probability of a low-quality access, since its higher associated SINR levels activate a more frequent usage of the less robust higher order modulation modes. For example, in conjunction with 400 users and two antenna array elements, $87 \%$ of LQ accesses occurred, while in the 16-QAM mode, with four array elements, this increased to $94 \%$. 


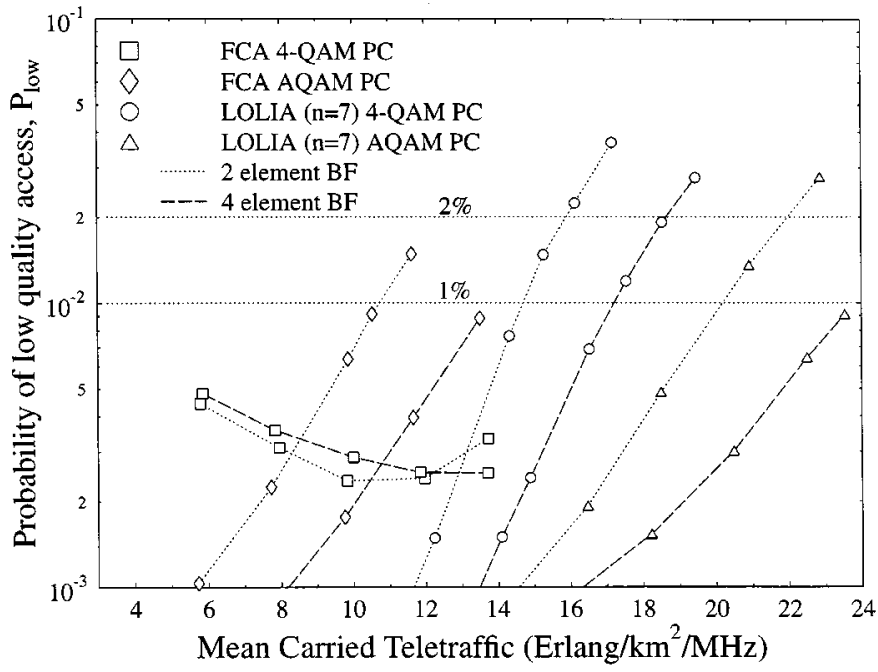

Fig. 10. Probability of low-quality access versus mean carried traffic of the LOLIA, with seven "local" base stations, and of FCA employing a seven-cell reuse cluster, for two- and four-element antenna arrays, with and without AQAM.

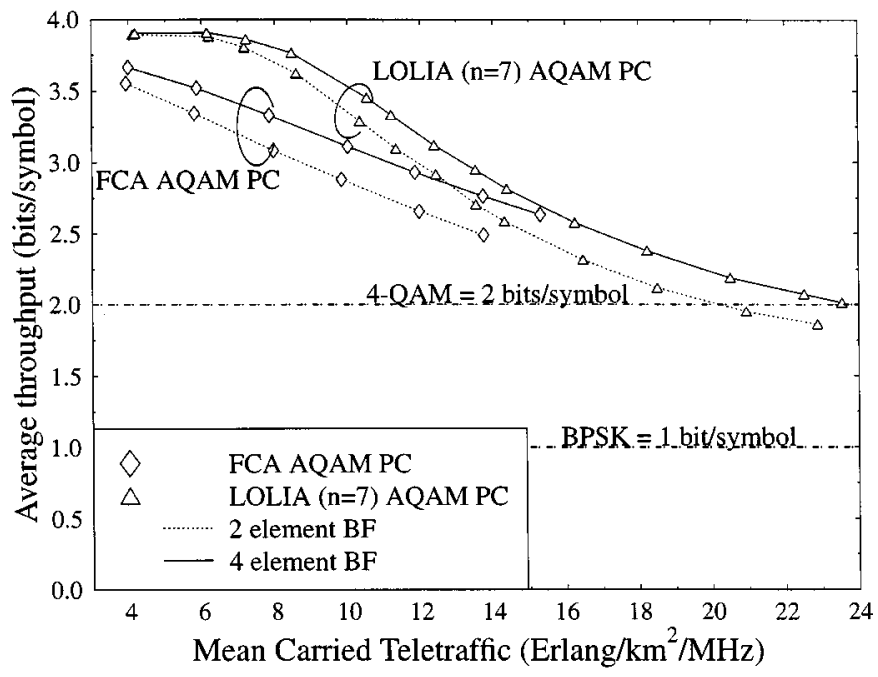

Fig. 11. Mean throughput of users in terms of bits per symbol versus mean carried traffic of the LOLIA, with seven "local" basestations, and of FCA employing a seven-cell reuse cluster, for two- and four-element antenna arrays, using AQAM.

However, as the network loading rises, an increasing proportion of the LQ outages occur in the BPSK modulation mode. Coupled with the increase in the BPSK modulation mode's usage due to the low SINR constraints, the probability of a low-quality outage must increase at a certain traffic load. This can be seen in Fig. 10, where the LQ outage probability is starting to rise for FCA with both two and four elements, though the extra interference suppression of the four-element array allows extra traffic to be carried, before this phenomenon commences. More specifically, for a network supporting 1200 users and using two-element adaptive antenna arrays, over $50 \%$ of the LQ accesses occurred, while in the 16-QAM mode, versus $74 \%$ with four-element antenna arrays. Increasing the number of users to 1400 reduced the number of 16-QAM LQ accesses, but increased the BPSK LQ outages to $60 \%$ and $24 \%$ for the two- and four-element arrays, respectively, with

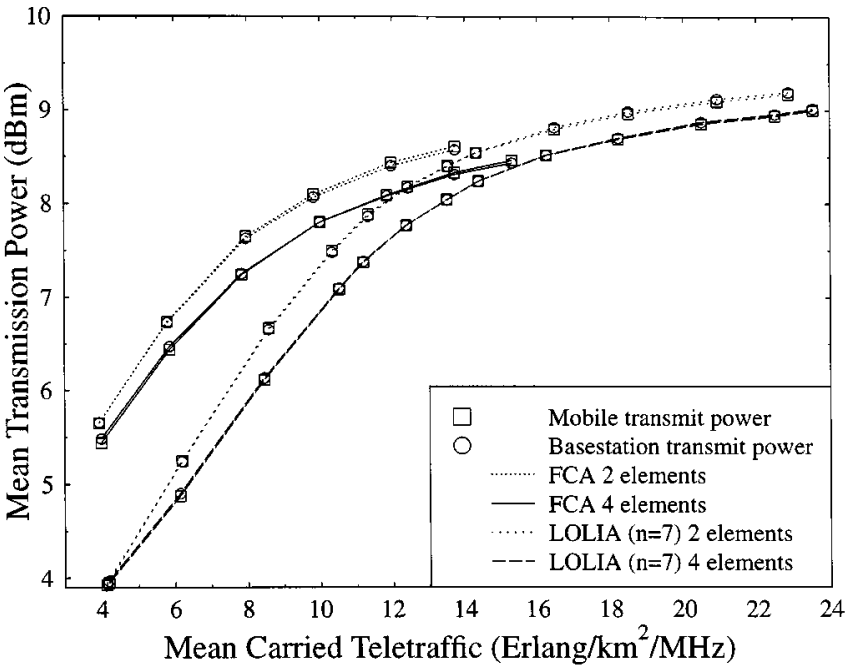

Fig. 12. Mean transmit power versus mean carried traffic of the LOLIA, with seven "local" base stations, and of FCA employing a seven-cell reuse cluster, for two- and four-element antenna arrays, using AQAM.

reductions to $28 \%$ and $48 \%$ of the LQ outages in the 16-QAM mode.

The average modem throughput expressed in bits per symbol versus the mean carried teletraffic is shown in Fig. 11. The figure shows that the mean number of bits per symbol decreased, as the network traffic increased. The FCA algorithm offered the least throughput and its performance degraded near-linearly with increasing network traffic. The LOLIA, especially for lower levels of traffic, offered a higher modem throughput for a given level of teletraffic carried, with the performance gracefully decreasing, as the carried teletraffic continued to increase.

The mean transmission power results of Fig. 12 demonstrate that the employment of AQAM reduced the power transmitted, both for the up- and the down-link. At low traffic levels, the FCA algorithm performed noticeably worse in transmitted power terms than the LOLIA. However, as the network traffic loading increased, the difference between the two algorithms was rapidly reduced. The mean power reduction, when compared to the fixed four-QAM transmission power of $10 \mathrm{dBm}$, varied from about 1 to more than $6 \mathrm{~dB}$ for the LOLIA. A $1-\mathrm{dB}$ reduction in the mean transmission power, from the $10-\mathrm{dBm}$ 4-QAM power, is not particularly significant for the mobile user, especially since at this network load a throughput of about 2 BPS is possible in both the four-QAM and AQAM scenarios. The difference between the network using AQAM and that without, though, is the overall improved call quality that can be achieved in the context of our performance metrics, and the significantly increased number of users that can be supported by the network.

\section{Summary of Results}

In order to arrive at a meaningful comparison of our results, it is necessary to consider a combination of the network performance metrics. For example, an algorithm may perform very well in one respect, yet have poor performance, when measured using an alternative metric. Therefore, the following conservative and lenient scenarios were defined [1]: 
TABLE II

MaXimum Mean Carried Traffic, and MaXimum Number of Mobile Users that Can Be Supported by Each Configuration, While MeEting the

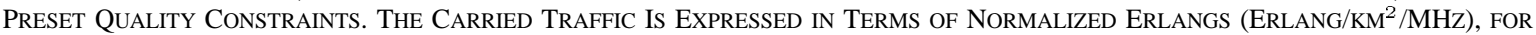
THE NETWORK DESCRIBED IN TABLE I IN A MULTIPATH ENVIRONMENT

\begin{tabular}{|c|c|c|c|c|c|c|}
\hline \multirow[t]{2}{*}{ Algorithm } & \multicolumn{3}{|c|}{$\begin{array}{c}\text { Conservative } \\
P_{F T}=1 \%, P_{\text {low }}=1 \% \\
G O S=4 \%, P_{B}=3 \%\end{array}$} & \multicolumn{3}{|c|}{$\begin{array}{c}\text { Lenient } \\
P_{F T}=1 \%, P_{\text {low }}=2 \% \\
G O S=6 \%, P_{B}=5 \%\end{array}$} \\
\hline & Users & Traffic & $\begin{array}{c}\text { Limiting } \\
\text { Factor }\end{array}$ & Users & Traffic & $\begin{array}{c}\text { Limiting } \\
\text { Factor }\end{array}$ \\
\hline \multicolumn{7}{|l|}{ 4-QAM } \\
\hline FCA, 2 element & 605 & 6.2 & $P_{\text {low }}$ & 780 & 7.9 & $P_{l o w}$ \\
\hline FCA, 4 elements & 850 & 8.7 & $P_{l o w}$ & 920 & 9.5 & $P_{F T}$ \\
\hline LOLIA $(n=7), 2$ element & 1235 & 12.9 & $P_{\text {low }}$ & 1330 & 14 & $P_{\text {low }}$ \\
\hline LOLIA $(\mathrm{n}=7), 4$ elements & 1415 & 14.6 & $P_{\text {low }}$ & 1525 & 15.9 & $P_{\text {low }}$ \\
\hline \multicolumn{7}{|l|}{ 4-QAM PC } \\
\hline FCA, 2 elements & 1060 & 10.3 & $P_{F T}$ & 1060 & 9.5 & $P_{F T}$ \\
\hline FCA, 4 elements & 1175 & 11.4 & $P_{F T}$ & 1175 & 11.4 & $P_{F T}$ \\
\hline LOLIA $(n=7), 2$ elements & 1440 & 14.7 & $P_{l o w}$ & 1530 & 15.9 & $P_{F T}$ \\
\hline LOLIA $(n=7), 4$ elements & 1665 & 17.2 & $P_{\text {low }}$ & 1815 & 18.7 & $P_{\text {low }}$ \\
\hline \multicolumn{7}{|l|}{ AQAM PC } \\
\hline FCA, 2 elements & 1160 & 11.6 & $P_{F T}$ & 1160 & 11.6 & $P_{F T}$ \\
\hline FCA, 4 elements & 1170 & 11.6 & $P_{F T}$ & 1170 & 11.6 & $P_{F T}$ \\
\hline LOLIA $(\mathrm{n}=7), 2$ elements & 1940 & 20.1 & $B P S / P_{\text {low }}$ & 1940 & 20.1 & $B P S$ \\
\hline LOLIA $(n=7), 4$ elements & 2300 & 23.6 & $B P S$ & 2300 & 23.6 & $B P S$ \\
\hline
\end{tabular}

- Conservative Scenario:

$P_{B} \leq 3 \%, P_{F T} \leq 1 \%, P_{\text {ow }} \leq 1 \%$ and GOS $\leq 4 \%$.

- Lenient Scenario:

$P_{B} \leq 5 \%, P_{F T} \leq 1 \%, P_{\text {low }} \leq 2 \%$ and GOS $\leq 6 \%$.

Due to the enhanced network performance resulting from the employment of AQAM, a further constraint of a minimum throughput of 2 BPS was invoked. This ensured a fair comparison with the fixed four-QAM-based network.

Table II shows how the networks using AQAM with power control, four-QAM with power control and four-QAM without power control, performed in terms of the number of users supported. A mean increase of $44 \%$ in the number of users was achieved by the addition of power control to the FCA algorithm-based four-QAM network. Invoking AQAM and power control led to a further average rise of $9 \%$, with any further gains limited by the nature of the call-dropping probability of the FCA algorithm. The interaction between the FCA algorithm and the AQAM-power control algorithm led to some interesting results, which suggest that an integrated channel allocation and adaptive modulation-power control algorithm used in conjunction with a smaller reuse distance would yield better results, especially with regard to the calldropping probability. The performance of the LOLIA was not limited in this way, however, and the addition of power control to the four-QAM network provided a mean increase of $17 \%$ extra users. In conjunction with AQAM techniques, this capacity was further extended by an average of $31 \%$, thus supporting an additional 54\% more users, when compared to the four-QAM network without power control.

\section{CONCLUSION}

In this paper, we have examined the performance of an AQAM-assisted mobile cellular network in contrast to a fixed four-QAM-based network. At high levels of network traffic, the network users benefited from improved call quality for a given transmit power and modem throughput. The network was thus capable of supporting more users with a higher user satisfaction than a similar network using fixed-mode four-QAM. For low numbers of network users, it offered superior call quality, reduced transmission powers, and higher modem throughput than a fixed four-QAM network in identical scenarios. Thus, for a given network traffic loading or number of users, our network employing AQAM was capable of achieving an increased mean modem throughput, while offering a better quality of service. Our future work involves the investigation of network capacity for a CDMA-based UMTS network.

\section{REFERENCES}

[1] P. Cherriman, F. Romiti, and L. Hanzo, "Channel allocation for thirdgeneration mobile radio systems," in Proc. ACTS, Rhodes, Greece, June 1998, pp. 255-260.

[2] A. Baiocchi, F. D. Priscoli, F. Grilli, and F. Sestini, "The geometric dynamic channel allocation as a practical strategy in mobile networks with bursty user mobility," IEEE Trans. Veh. Technol., vol. 44, pp. 14-23, Feb. 1995.

[3] F. D. Priscoli, N. P. Magnani, V. Palestini, and F. Sestini, "Application of dynamic channel allocation strategies to the GSM cellular network," IEEE J. Select. Areas Commun., vol. 15, pp. 1558-1567, Oct. 1997.

[4] J. Blogh, P. Cherriman, and L. Hanzo, "Adaptive beamforming assisted dynamic channel allocation," in Proc. VTC, Houston, TX, May 1999, pp. 199-203. 
[5] — "Dynamic channel allocation using adaptive antennas and power control," in Proc. ACTS Summit, Sorrento, Italy, June 1999, pp. 943-948.

[6] A. Goldsmith and S. Chua, "Variable rate variable power MQAM for fading channels," IEEE Trans. Commun., vol. 45, pp. 1218-1230, Oct. 1997.

[7] W. Webb and R. Steele, "Variable rate QAM for mobile radio," IEEE Trans. Commun., vol. 43, pp. 2223-2230, July 1995.

[8] S. Sampei, S. Komaki, and N. Morinaga, "Adaptive modulation/TDMA scheme for large capacity personal multimedia communications systems," IEICE Trans. Commun., vol. E77-B, pp. 1096-1103, Sept. 1994

[9] L. C. Godara, "Applications of antenna arrays to mobile communications-Part I: Performance improvement, feasibility, and system considerations," Proc. IEEE, vol. 85, pp. 1029-1060, July 1997.

[10] J. Litva and T. Lo, Digital Beamforming in Wireless Communications. London, U.K.: Artech House, 1996.

[11] L. C. Godara, "Applications of antenna arrays to mobile communications-Part II: Beam-forming and direction-of-arrival considerations," Proc. IEEE, vol. 85, pp. 1193-1245, Aug. 1997.

[12] T. S. Rappaport, Ed., Smart Antennas: Adaptive Arrays, Algorithms and Wireless Position Location. Piscataway, NJ: IEEE, 1998.

[13] I. Reed, J. Mallett, and L. Brennan, "Rapid convergence rate in adaptive arrays," IEEE Trans. Aerosp. Electron. Syst., vol. AES-10, pp. 853-863, Nov. 1974.

[14] R. Monzingo and T. Miller, Introduction to Adaptive Arrays. New York: Wiley, 1980.

[15] D. Gerlach and A. Paulraj, "Adaptive transmitting antenna arrays with feedback,” IEEE Signal Processing Lett., vol. 1, pp. 150-152, Oct. 1994.

[16] _ _ "Base station transmitting antenna arrays for multipath environments," Signal Process., pp. 59-73, 1996.

[17] L. Hanzo, W. T. Webb, and T. Keller, Single-and Multi-Carrier Quadrature Amplitude Modulation. New York: Wiley/IEEE, 2000.

[18] J. M. Torrance, L. Hanzo, and T. Keller, "Interference aspects of adaptive modems over slow Rayleigh fading channels," IEEE Trans. Veh. Technol., vol. 48, pp. 1527-1545, Sept. 1999.

[19] J. M. Torrance and L. Hanzo, "Latency and networking aspects of adaptive modems over slow indoors Rayleigh fading channels," IEEE Trans. Veh. Technol., vol. 48, pp. 1237-1251, July 1999.

[20] C. H. Wong and L. Hanzo, "Upper-bound performance of a wideband burst-by-burst adaptive mode," IEEE Trans. Commun., vol. 48, pp. 367-369, Mar. 2000.

[21] M. M. L. Cheng and J. C. I. Chuang, "Performance evaluation of distributed measurement-based dynamic channel assignment in local wireless communications," IEEE J. Select. Areas Commun., vol. 14, pp. 698-710, May 1996.

[22] A. S. Tanenbaum, "Introduction to queueing theory," in Computer Networks, 2nd ed. Englewood Cliffs, NJ: Prentice-Hall, 1989, pp. 631-641.

[23] J. C. I. Chuang and N. R. Sollenberger, "Performance of autonomous dynamic channel assignment and power control for TDMA/FDMA wireless access," IEEE J. Select. Areas Commun., vol. 12, pp. 1314-1323, Oct. 1994.

[24] P. Cherriman and L. Hanzo, "Programmable H.263-based wireless video transceivers for interference-limited environments," IEEE Trans. Circuits Syst. Video Technol., vol. 8, pp. 275-286, June 1998.

[25] J. Liberti and T. Rappaport, "A geometrically based model for line-ofsight multipath radio channels," in Proc. VTC , 1996, pp. 844-848.

[26] R. Ertel, P. Cardieri, K. Sowerby, T. Rappaport, and J. Reed, "Overview of spatial channel models for antenna array communications systems," IEEE Pers. Commun., vol. 5, pp. 10-22, Feb. 1998.
[27] R. Kohno, Wireless Communications: TDMA Versus CDMA. Boston, MA: Kluwer, 1997, ch. 1, pp. 293-321.

[28] Y. Ogawa and T. Ohgane, "Adaptive antennas for future mobile radio," IEICE Trans. Fund., vol. E79-A, pp. 961-967, July 1996.

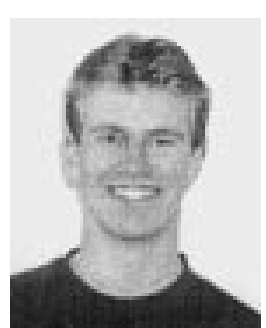

Jonathan S. Blogh received the M.Eng. degree in 1997 (with distinction) in information engineering from the University of Southampton, Southampton, U.K. Since 1997, he has been pursuing the Ph.D. degree in mobile communications at the University of Southampton.

His current areas of research include the networking aspects of FDD and TDD mode third-generation mobile cellular networks.

Mr. Blogh was awarded the IEE Lord Lloyd of Kilgerran Memorial Prize for interest and commitment to mobile radio and RF engineering in 1997

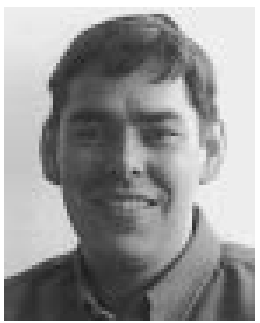

Peter J. Cherriman received the M.Eng. degree in information engineering from the University of Southampton, Southampton, U.K. in 1994. Since 1994, he has been pursuing the Ph.D. degree in mobile video networking with the Department of Electronics and Computer Science at the University of Southampton.

Currently he is working on projects for the Mobile Virtual Centre of Excellence. His current areas of research include robust video coding, microcellular radio systems, power control, dynamic channel allocation, and multiple access protocols.

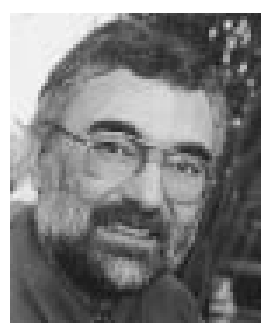

Lajos Hanzo (SM'92) received the Dipl. Ing. degree in electronics in 1976 and the Ph.D. degree in 1983, both from the Technical University of Budapest, Hungary

During his 24-year career in telecommunications, he has held various research and academic posts in Hungary, Germany, and the U.K. Since 1986, he has been with the Department of Electronics and Computer Science, University of Southampton, Southampton, U.K., and has been a Consultant to Multiple Access Communications Ltd., Southampton, U.K. Currently he holds a chair in telecommunications. He co-authored five books on mobile radio communications, published about 300 research papers, organized and chaired conference sessions, presented overview lectures, and was awarded a number of distinctions. Currently he is managing an academic research team, working on a range of research projects in the field of wireless multimedia communications sponsored both by industry and under the auspices of the Engineering and Physical Sciences Research Council (EPSRC), U.K., the European IST Programme, and the Mobile Virtual Centre of Excellence (VCE), U.K. For further information on research in progress and associated publications, please refer to http://www-mobile.ecs.soton.ac.uk. 